\title{
DEFINICIÓN DE UNA NUEVA FASE CONSTRUCTIVA EN HUACA PUCLLANA
}

\author{
MIRELLA GANOZA YAIPÉN ${ }^{1}$ \\ PROYECTO DE INVESTIGACIÓN, CONSERVACIÓN Y PUESTA EN VALOR HUACA PUCLLANA \\ mganoza@gmail.com
}

\section{RESUMEN}

En la sexta plataforma del Centro Ceremonial Huaca Pucllana se ha encontrado elementos que por sus características formales y tecnología empleada correspondería a una fase de ocupación, para el caso de Huaca Pucllana se trataría de la Fase Constructiva IV, que complementaría la información sobre el diseño y crecimiento arquitectónico de la Gran Pirámide y su superposición sobre las fases anteriores.

Palabras Claves: sexta plataforma, arquitectura, fase constructiva.

\section{Abstract}

In the Ceremonial Center Huaca Pucllana, at the sixth platform, it found elements by their formal features and technology used correspond to a phase of occupation; in the case of Huaca Pucllana would be the Construction Phase IV, which would complement the design information and architectural growth of the Great Pyramid and its superposition on the previous phases.

KeYwoRDs: sixth platform, architecture, phase occupation.

1. Licenciada en Arqueología. Universidad Nacional Mayor de San Marcos. 


\section{GENERALIDADES}

El sitio arqueológico Huaca Pucllana se encuentra ubicado en el distrito de Miraflores, provincia de Lima; tiene una extensión actual de 6ha. Este asentamiento se compone de una pirámide escalona$\mathrm{da}$, compuesta por siete plataformas, tiene $500 \mathrm{~m}$ de largo por $80 \mathrm{~m}$ de ancho y $25 \mathrm{~m}$ de alto y en la parte baja, anexada a la pirámide, ocupando el noreste del área se tienen construcciones menores. Fue un centro ceremonial importante durante los siglos II d.C al VI d.C; esta construcción se fue edificando y remodelando continuamente con sucesivas construcciones logrando así la altura y volumen que tiene en la actualidad (Flores, 2005; 2013c). El sitio arqueológico viene siendo estudiado desde el año 1981 por el Proyecto de Investigación, Conservación y Puesta en Valor Huaca Pucllana (PICPVHP) a cargo de la Dra. Isabel Flores Espinoza. (Figura 1)

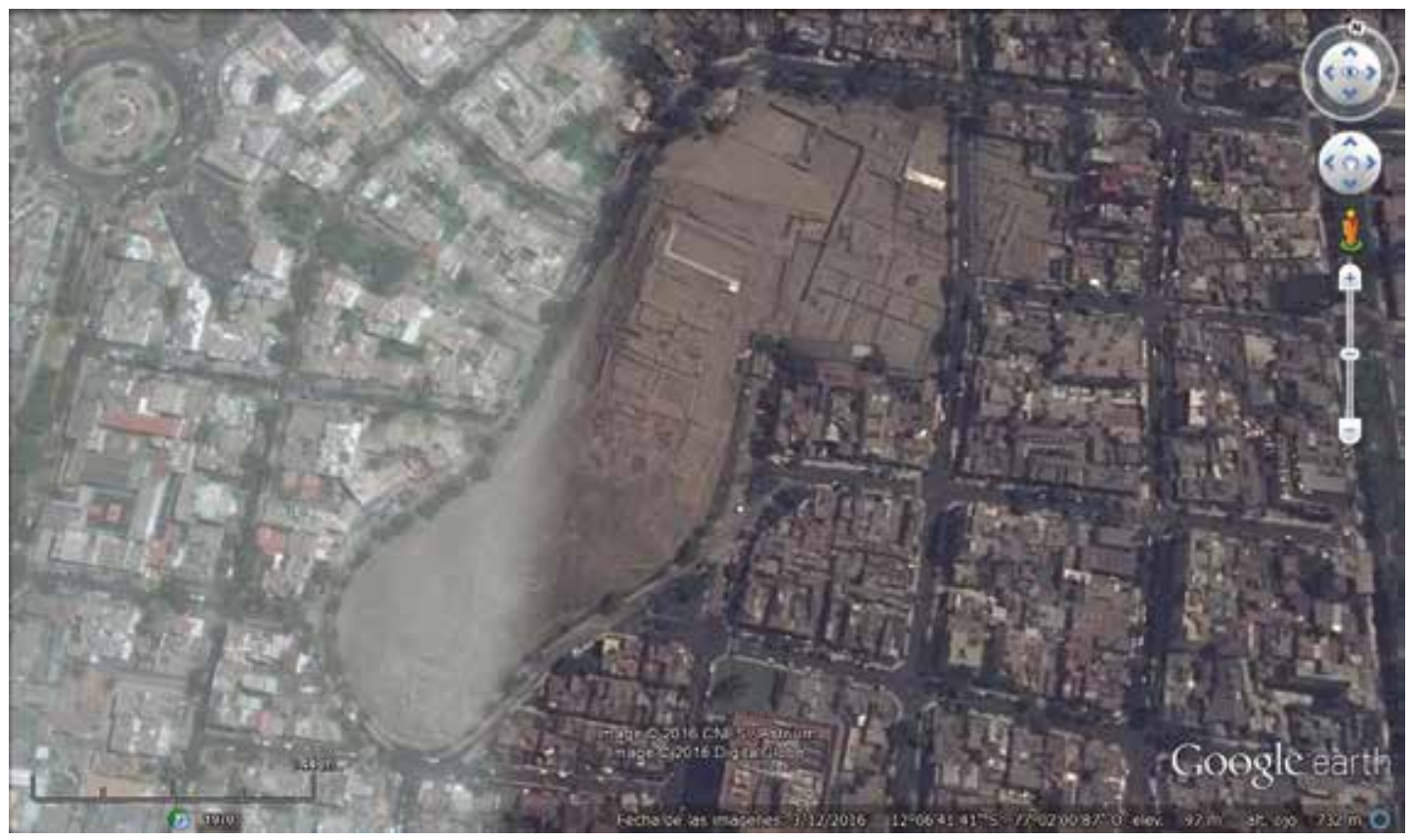

Figura 1.

Si observamos lo que es la arquitectura, ésta de por si surge cuando el espacio empieza a ser conformado y estructurado por los diferentes elementos físicos que lo conforman; teniendo en cuenta que el concepto de arquitectura es muy amplio, desde definirse como espacios conformados por diferentes elementos arquitectónicos, hasta de considerarse como espacios para diferentes actividades relacionadas al quehacer del ser humano (protección, servicio, consumo, ceremonias, etc.); entonces se concibe a las construcciones arquitectónicas, ya sean edificios públicos, ceremoniales o de cualquier otro tipo como construcciones culturales cargadas de símbolos y además como un instrumento del uso de poder, asimismo nos informan sobre el orden social del grupo de lo construyó (traducido de Moore, 1996: 2). La línea de investigación de Arqueología de la Arquitectura entiende por "Arquitectura, la manipulación antrópica de un espacio dado mediante técnicas constructivas que varían a lo largo del tiempo atendiendo a factores sociales, culturales y económicos. La arquitectura estaría relacionada tanto con su entorno físico como con la sociedad que la genera, siendo su forma concreta fruto de una idea o percepción compartida por la colectividad de individuos de una sociedad y por lo 
tanto comprensible dentro de ella, directamente relacionada con los códigos de uso y concepción del espacio y con los esquemas de pensamiento de esa sociedad (Mañana, et al. 2002: 14). Además las construcciones pasan a su vez por numerosas modificaciones de distinta índole; desde problemas estructurales, hasta por cambios culturales; el análisis o estudio de estos cambios nos ayudan a acercarnos un poco más al entendimiento del grupo social, el cual ha depositado sus esquemas de vida en dichas construcciones, siendo importante además identificar la forma inicial de las construcciones como las variaciones que han sufrido a lo largo del tiempo (Mañana, et al. 2002: 18).

En Huaca Pucllana se han registrado, hasta el momento, tres grandes momentos de cambios arquitectónicos, estos han sido señalados como "fases constructivas", las cuales se sustentan en el cambio de los elementos usados en la construcción de las edificaciones, estando esto bien diferenciado para las tres fases ya definidas, estas fases se encuentran superpuestas arquitectónicamente y diferenciadas cronológicamente. De igual manera los cambios ocurridos dentro de cada fase constructiva se conocen como etapas, estas se refieren a un cambio dentro del diseño arquitectónico del espacio, pero con las características de la fase a la que pertenece: Fase I, construcciones en técnica de tapial; los rellenos arquitectónicos son bloques de barro y cantos rodados. Fase II, construcciones en adobes cúbicos y paralelepípedos cuadrangulares $(16 \times 16 \times 9 \mathrm{~cm})$; los rellenos arquitectónicos son adobes cúbicos y odontiformes. Fase III, construcciones de adobes paralelepípedos rectangulares $(21 \times 14 \times 7 \mathrm{~cm})$, uso de pintura amarilla en los muros; los rellenos arquitectónicos son principalmente cantos rodados y arena gris (Flores 2005: 54).

Los trabajos de excavación realizados en la sexta plataforma desde el año 2009 hasta el 2015 abarcan un área de $820 \mathrm{~m} 2$ aproximadamente, que corresponde al $40 \%$ del área total. En estos trabajos se observó un cambio en la tecnología constructiva representada en los diferentes elementos arquitectónicos: elaboración de muros, pisos y banquetas, orientación de los muros, uso de los espacios, etc. Es importante mencionar que en la quinta plataforma se ha encontrado arquitectura de la Fase Constructiva IV en su extremo sur; en esta plataforma se conoce la existencia de dos sacrificios humanos ubicados en un relleno arquitectónico y asociados a rituales de cambios drásticos de arquitectura, asimismo estos entierros se asocian al relleno sobre el cual se construyó un muro que pertenecería a la última etapa de ocupación propuesta, por el material cultural recuperado (cerámica Pucllana Nievería tardía) se relaciona al Horizonte Medio 1B (Barreto, et. al. 2010)ํ․

\section{Antecedentes de los Trabajos Realizados en la Sexta Plataforma}

La sexta plataforma es el nivel más elevado y el último en construirse en Huaca Pucllana, la cual se encuentra aún en proceso de excavación. Esta plataforma contiene la información sobre los cambios que suceden en los últimos momentos de ocupación de la sociedad Lima previo al abandono definitivo del Centro Ceremonial. Las excavaciones arqueológicas muestran diferencias relacionada a la técnica constructiva; al observar y analizar los datos se identificó un cambio en la tecnología, métodos y procesos utilizados para llevar a cabo las construcciones, en comparación con las precedentes (específicamente la Fase Constructiva III).

Los trabajos de excavación en la sexta plataforma se habían limitado inicialmente a las limpiezas superficiales y principalmente a excavación de áreas con presencia de tumbas intrusivas Wari, se ha mencionado lo siguiente:

Flores (2012a) al hacer un resumen de las ocupaciones en Huaca Pucllana, menciona que “... en la pirámide luego del apogeo reflejado por varias remodelaciones donde mantiene una unidad constructiva, en las ultimas construcciones empieza a producirse un cambio paulatino que finaliza con 
un cambio radical y su posterior abandono, los cambios consisten en construcción de ambientes más pequeños en donde los muros se elaboran más delgados y ya no son pintados de color amarillo. Estos cambios también son notorios en la cerámica, al transcurrir el tiempo aparece un alfar mucho más fino de color anaranjado, al cual se le está denominando Alfar Pucllana Nievería; solamente las ultimas construcciones de Pucllana estarían relacionadas con la cerámica del estilo Nievería; y de acuerdo a la ubicación propuesta por Dorothy Menzel correspondería a la Época 1B del Horizonte Medio. Luego de esto se daría el abandono del sitio, el cual duró aproximadamente 100 años" (15-18).

Flores, et al. (2012b) al describir los patios con banqueta ubicados en el extremo noreste de la sexta plataforma (Patios 16 y 22), se menciona que estos son considerados como construcciones de la etapa tardía de la fase constructiva III. Se indican las características físicas de los muros y del piso (dimensiones, ausencia de pintura amarilla, falta de elaboración, etc.), además se señala que los rellenos están compuestos por adobes enteros y fragmentados, pareciendo ser restos de demolición, se encuentran rellenos similares en otras zonas de la sexta plataforma; estas características se asociarían al Horizonte Medio $1 \mathrm{~B}$ por ubicarse a la misma altura que construcciones en la quinta plataforma donde se ha encontrado cerámica del tipo "Nievería B". Estas construcciones eran consideradas las únicas unidades arquitectónicas de la última etapa constructiva en Huaca Pucllana (79-81).

Vargas (2012) al proponer la secuencia constructiva de la Fase Constructiva III en la Gran Pirámide, y prestando atención a las dos últimas etapas constructivas (Etapa Pampilla y Etapa Piedritas) menciona: "se observa un cambio aparentemente sutil pero de importancia en lo ideológico", se da la eliminación de los muros pintados de amarillo, se tiene un solo patio con banqueta y se produce una disminución de restos de desechos orgánicos sobre el piso, cerámica o actividad sacrificial. Se propone además un cambio en la funcionalidad del sitio, por relacionarse con la aparición de la cerámica con diseños de la costa y sierra sur vinculado a Wari. Se indica la presencia de muros angostos, de poca altura, construidos con adobes reutilizados; los rellenos son de adobes, restos de demolición, y sobre estos se construyen muros con adobes reciclados; estas construcciones se asocian a la cerámica del estilo Nievería. Luego de esto se da el abandono del sitio (308-309).

Flores (2013b) en la descripción de las ocupaciones y las plataformas que conforman el Centro Ceremonial se menciona "El sexto nivel es el más alto. Es de forma alargada y ha sido poco excavado. La arquitectura hallada era compleja debido a la fuerte destrucción por la presencia de tumbas Wari, pues la mayor parte fue alterada para la elaboración de profundas tumbas. Al parecer en este nivel también hubo patios con banquetas" (25-27).

Si bien son pocas las referencias acerca la arquitectura propia a la ocupación de la sexta plataforma, todas estas asociaban la ocupación a la etapa tardía de la fase constructiva III, así como ocupaciones finales Lima inmediatamente previas al abandono, sin embargo estos cambios arquitectónicos no fueron un solo evento, sino una serie de cambios ocurridos en un espacio de tiempo determinado.

De los antecedentes mencionados se tienen los siguientes puntos como indicadores de cambio constructivo y arquitectónico:

- Muros delgados, elaborados en su mayoría con adobes reutilizados.

- Pisos de poco grosor y mal elaborados.

- Rellenos de adobes enteros y fragmentados, restos de demolición de construcciones previas.

- Ausencia de pintura amarilla en los muros.

- Presencia de cerámica Nievería, sobre todo el alfar Pucllana Nievería tardío (o estilo Nievería, propuesto por Menzel). 


\section{Definiendo los Indicadores de la Fase Constructiva IV.}

Teniendo en cuenta que la variable principal para la definición de la cuarta fase constructiva es la arquitectura, entonces la caracterización de los distintos indicadores arquitectónicos ayudan a comprender y exponer los cambios ocurridos en la fase estudiada. Los principales son:

Los muros, se utiliza adobes paralelepípedos alargados $(19.5 \times 13 \times 6.5 \mathrm{~cm})$, nuevos y reutilizados, producto del desmontaje de anteriores construcciones, se construyen con menor ancho que los de la fase anterior, en la fase constructiva III los muros tiene un ancho aproximado de $1.5 \mathrm{~m}$, en la primera etapa de la fase IV tienen aproximadamente $1.00 \mathrm{~m}$ a $1.15 \mathrm{~m}$ y en la última etapa llegan a tener entre $0.45 \mathrm{~m}$ a $0.50 \mathrm{~m}$ de ancho, mostrando una notable reducción en el ancho de los muros; los adobes de estos muros algunas veces poseen restos de pintura amarilla, por ser reutilizados de muros de la fase anterior. Estos muros son construidos sobre una capa de tierra y/o relleno de terrones de barro, a diferencia de los muros de la fase anterior que se elaboran directamente sobre el piso; algunos muros son elaborados sobre uno anterior, para el cual se ha desmontado y vuelto a edificar con un pequeño cambio en su orientación. Los paramentos de los muros presentan una delgada capa de enlucido color beige de $0.5 \mathrm{~cm}$ de grosor y totalmente carente de pintura amarilla. (Figura 2)
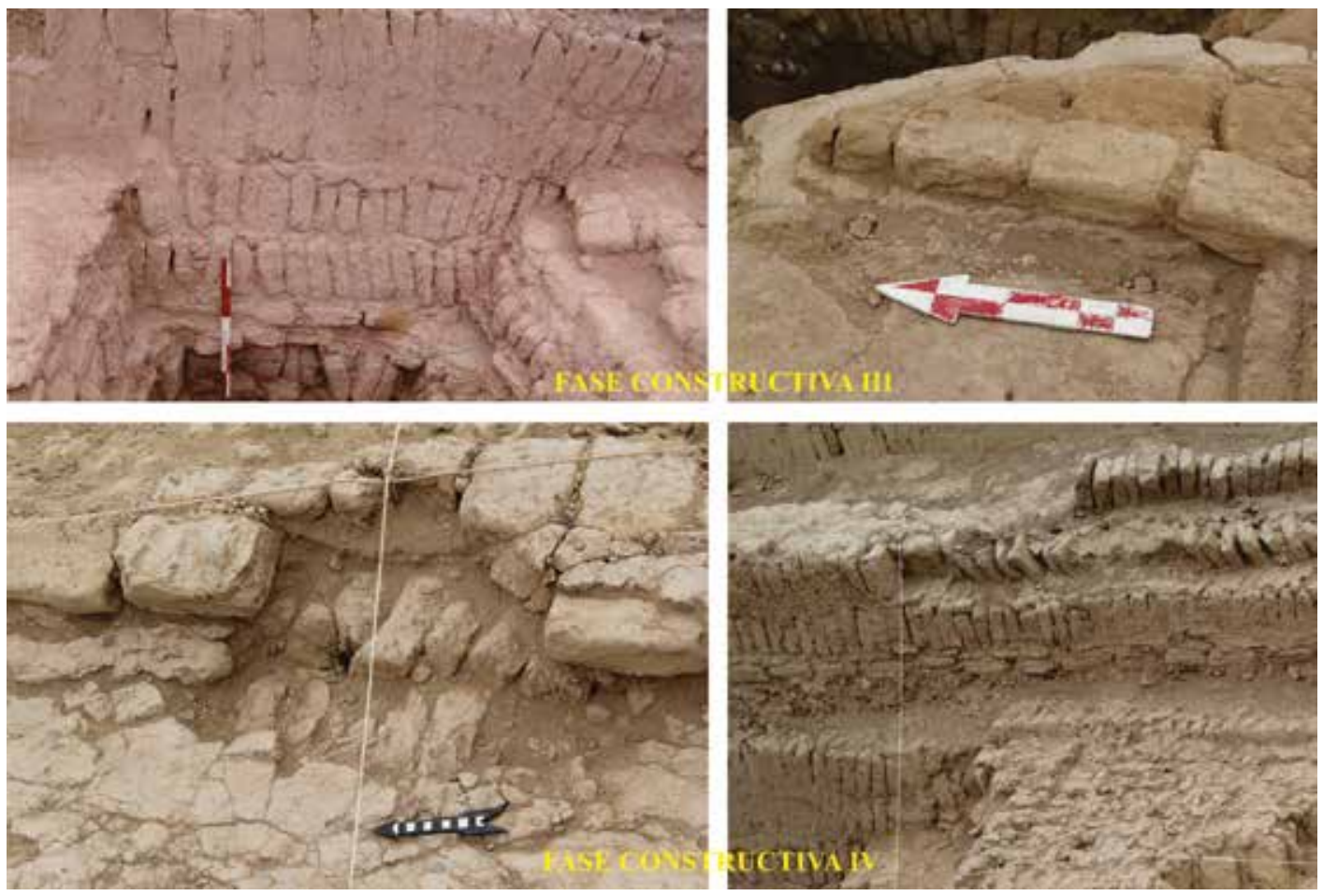

Figura 2. (superior) y Figura 3. (inferior)

Los pisos, no son bien elaborados, utilizan menor cantidad de barro, llegan a tener poco grosor y sin mayor acabado superficial, a pesar de realizarse varias remodelaciones en ellos; el grosor de los pisos pueden tener entre 1 a $3 \mathrm{~cm}$ de espesor; a diferencia de los pisos de la fase constructiva III, estos llegan a tener hasta $10 \mathrm{~cm}$ de grosor, siendo capas compactas de barro y presentando un acabado superficial más elaborado. (Figura 3) 

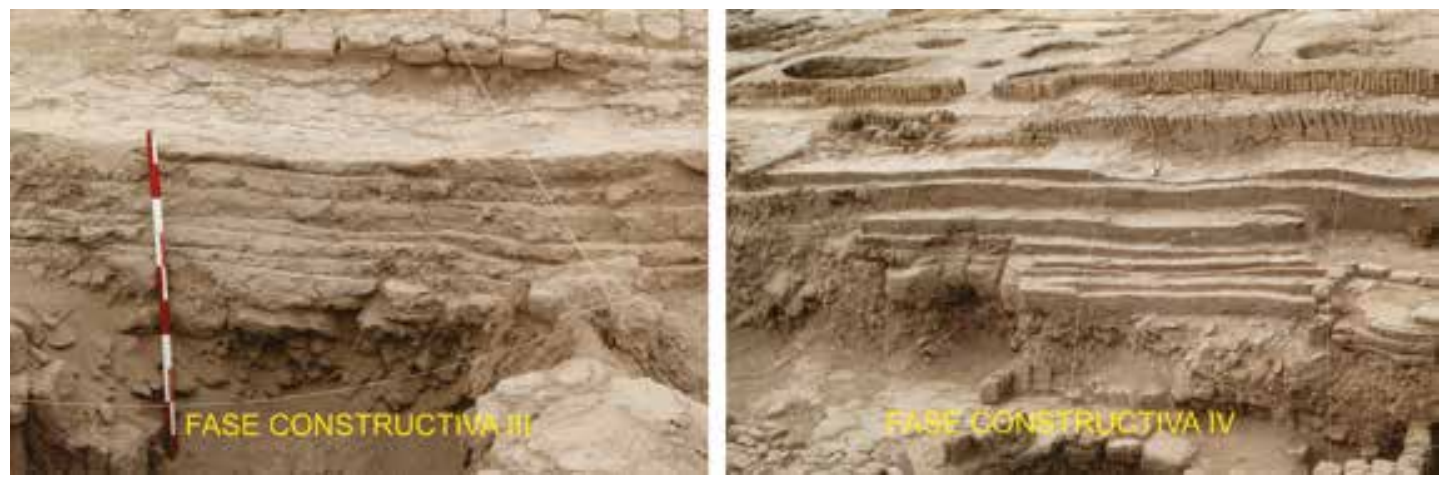

Figura 4.

Los rellenos arquitectónicos los cuales son utilizados para cubrir la superficie de los distintos ambientes, son depósitos de tierra y adobes enteros y fragmentados principalmente, la presencia de los adobes son producto de la destrucción de construcciones, en comparación con los rellenos de la fase anterior, que suelen usar depósitos de cantos rodados mezclados con arena gris y tierra. (Figura 4)

\begin{tabular}{|c|c|c|}
\hline \multicolumn{3}{|c|}{ INDICADORES ARQUITECTÓNICOS } \\
\hline \multirow{2}{*}{$\begin{array}{l}\text { ELEMENTOS } \\
\text { ARQUITECTÓNI } \\
\text { COS }\end{array}$} & \multicolumn{2}{|c|}{ FASES CONSTRUCTIVAS } \\
\hline & F.C. III & F.C. IV \\
\hline \multirow{3}{*}{ Pisos } & Pisos gruesos ( $5 \mathrm{~cm}$ a mas) & Pisos delgados (1-3cm) \\
\hline & Consistencia muy compacta & Consistencia compacta \\
\hline & Buen acabado superficial & Poco acabado superficial \\
\hline \multirow{3}{*}{ Muros } & $\begin{array}{l}\text { Consutruidos directamente } \\
\text { sobre la superficie del piso }\end{array}$ & $\begin{array}{l}\text { Elaborados sobre capa de } \\
\text { tierra o rellenos (destruccion } \\
\text { del muro anterior) }\end{array}$ \\
\hline & $\begin{array}{l}\text { Enlucido frueso }(1 \mathrm{~cm} \mathrm{de} \\
\text { espesor) }\end{array}$ & $\begin{array}{l}\text { Capa delgada de enlucido } \\
(0.5 \mathrm{~cm} \text { de espesor })\end{array}$ \\
\hline & Presencia de pintura amarilla & Ausencia de pintura amarila \\
\hline Rellenos & $\begin{array}{l}\text { Principalmente cantos } \\
\text { rodados mezclados con arena } \\
\text { gris }\end{array}$ & $\begin{array}{l}\text { Adobes enteros y } \\
\text { fragmentados (producto de } \\
\text { destruccion de } \\
\text { consitrucciones anteriores) }\end{array}$ \\
\hline
\end{tabular}

El material cultural recuperado, sobre todo la cerámica, ha servido de material comparativo para determinar la temporalidad de las construcciones. Si bien la cerámica no ha sido abundante, notamos la presencia del alfar Pucllana Nievería de manera muy escasa en la primera etapa constructiva y luego aumenta la cantidad de fragmentos y además de notar la presencia en la última etapa del alfar Pucllana Nievería Gris² (Ccencho, 2006: 19).

\section{Descripción de las Etapas Constructivas de la Fase Constructiva IV.}

Una fase constructiva puede encontrarse dividida en etapas de acuerdo al cambio en el diseño del espacio (Flores, 2005: 56), en esta fase se ha identificado hasta el momento tres etapas, donde se aprecia como los espacios van siendo modificados. Se ha identificado tres etapas constructivas, denominadas: Temprana, Media y Tardía (Flores, 2013a: 102-111; 2013c: 25, 26). En estas se muestra como los espacios van siendo modificados, pasando de ambientes de grandes dimensiones, a recintos de menor tamaño y con diferente uso, pero sin dejar de lado espacios importantes como los patios con banquetas, que son los espacios con mayor jerarquía registradas en Huaca Pucllana y parte principal 
de los espacios públicos donde se materializan las jerarquías sociales a través de la exposición de los distintos estamentos sociales en determinadas reuniones (Flores et al. 2012b: 59); estos cambios estarían respondiendo a cambios dentro de la estructura del grupo social que lo edificó. Es así que cada etapa constructiva se conforma por una o más unidades arquitectónicas (UA).

Etapa Temprana: La arquitectura de esta etapa se define por un gran espacio o un patio muy amplio (Flores 2013a: 102-103), este patio ha sido identificado como UA-08(12) ${ }^{3}$ (Figura 5), la cual corresponde a un patio de planta rectangular de grandes dimensiones, el cual se puede observar el piso de este espacio a lo largo del perfil estratigráfico del lado norte, está unidad aún no ha sido excavada completamente, solo se ha conservado el extremo sur del mismo casi del ancho de la sexta plataforma. Presenta los muros que lo delimitan hacia el Sur [MU-14(12)] y al Este [MU-16(12)], tendrían un grosor aproximado de $1.15 \mathrm{~m}$, los que estarían formando la esquina sureste del recinto; estos muros y sobre todo el muro Este se estarían elaborando sobre la destrucción de un muro de la fase anterior, tendrían casi la misma orientación, pero con una ligera desviación hacia el NE. Estos muros están asociados a un piso [PI-19(12)], el cual solo tiene $2 \mathrm{~cm}$ de espesor, además se encontró muy desgastado por las actividades que se realizaron al interior y probablemente al clausurar el ambiente, como la ampliación de los hoyos de poste para su retiro, los cuales se encuentran alineados al MU-16(12) (Este). También se ha registrado áreas de preparación de barro y presencia de hoyos pequeños al parecer de carácter ritual ${ }^{4}$ (Flores, 2005; Silvera, 2012) distribuidos casi al centro del recinto. Este recinto debió de extenderse hacia el norte y debió de asociarse a otros espacios tanto al sur como al oeste de la sexta plataforma, pero que aún no se han excavado y se encuentran cubiertas por las construcciones de las demás etapas. La clausura del espacio se dio utilizando dos tipos de rellenos superpuestos; el primero se trató de un relleno de adobes, enteros y fragmentados, que se distribuye en todo el piso del ambiente, se encontraron cinco hoyos pequeños de carácter ritual, en su interior fragmentos pequeños de adobes quemados, un fragmento pequeño de batán, una aguja, dumortierita y ofrendas de ave. El segundo relleno es un relleno de tierra grumosa de consistencia semicompacta y cubre el depósito anterior y por sus características debió tratarse de tierra agrícola, colocada semi húmeda y con escaso material cultural.

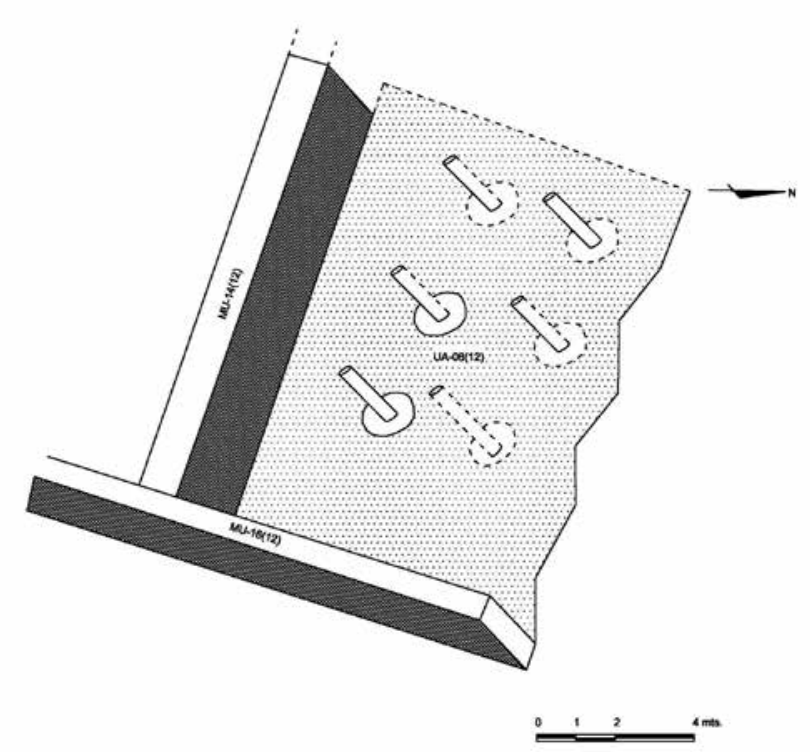

Figura 5. 
Etapa Media: De lo excavado se puede decir que está constituido por dos unidades arquitectónicas contiguas y contemporáneas, estos espacios son: UA-03(12) y UA-04(12), ambas divididas por el MU14(12), el cual es el muro que se reutiliza de la etapa anterior (Flores, 2013a; Ganoza, 2015). (Figura 6)

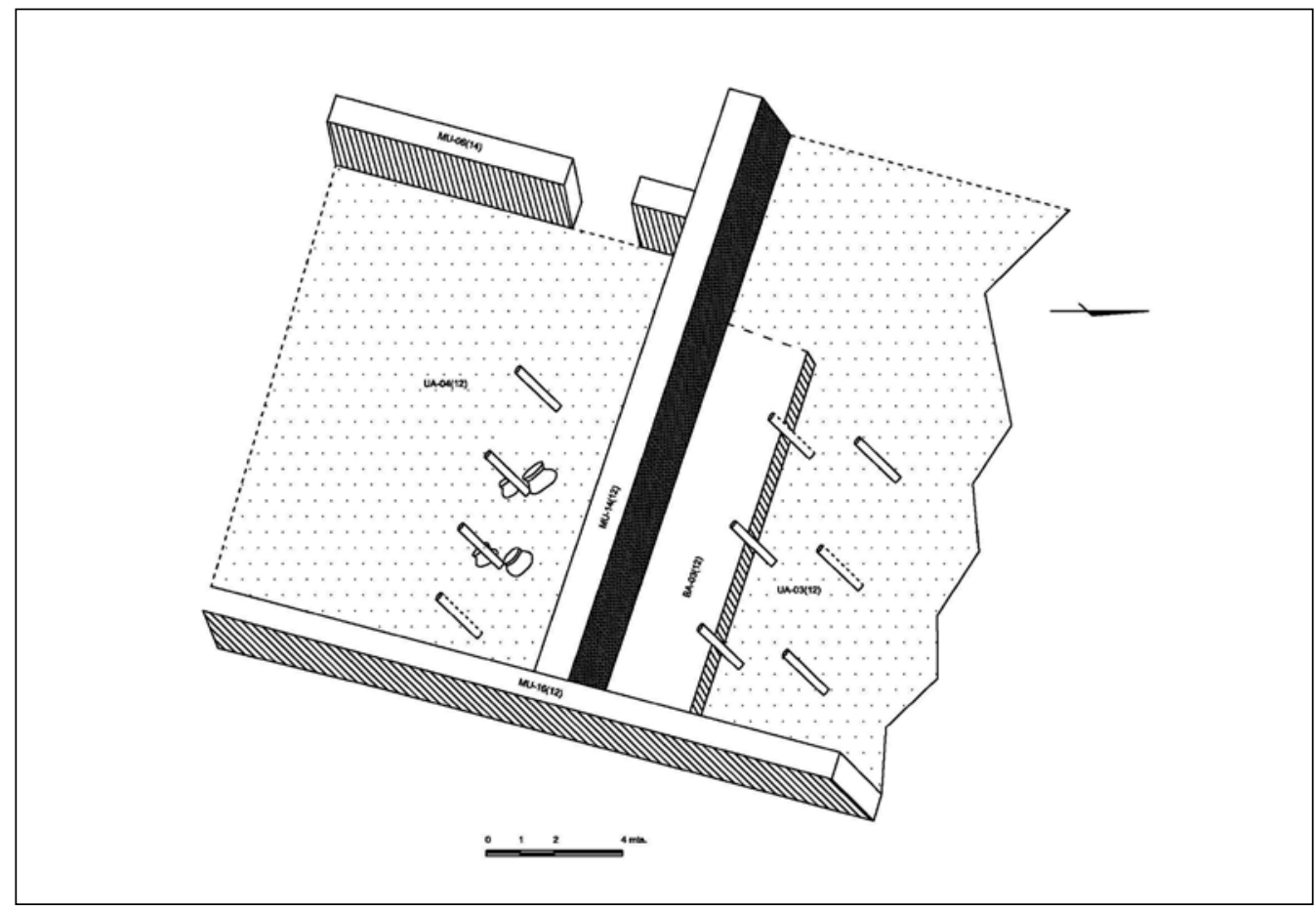

Figura 6.

La UA-03(12) se superpone a la UA-08(12) de la etapa anterior, se trata de un recinto del tipo patio con banqueta [BA-03(12)], la cual se encuentra ubicada al sur del ambiente, teniendo como respaldar sur al MU-14(12). En este ambiente se han encontrado hoyos de postes alineados a la banqueta, sobre el piso se halló una capa delgada y compacta de tierra mezclada con restos orgánicos (ictiológico y vegetal), así como tres evidencias de quema; asociados al piso y en la parte central se encontraron hoyos pequeños de carácter ritual. La UA-04(12) se encuentra al sur de la UA-03(12) y como ya se mencionó se encuentran separadas por el MU-14(12), hacia el Oeste se tiene el MU-06(14), donde además se forma el vano de acceso a la unidad arquitectónica. Este ambiente corresponde a un patio donde se encontraron hoyos de postes y también improntas de vasijas de forma circular y fondo cóncavo, además se observan pequeñas áreas de quema con basura mezclada con restos orgánicos, siendo indicador de una actividad que se realizaba en este ambiente, al parecer serían actividades de consumo y preparación de alimentos, los cuales podrían estar relacionados con lo que sucedía en la unidad arquitectónica adyacente. Asociado a la elaboración del piso se encontraron hoyos pequeños de carácter ritual, en uno de ellos se descubrió un fragmento pequeño de madera, con el que tal vez debió de elaborarse dichos hoyos. Para la clausura de esta etapa se colocó un relleno de tierra mezclada con adobes (enteros y fragmentados), estos adobes deben corresponder a la destrucción de elementos arquitectónicos, algunos de los adobes encontrados tuvieron restos de pintura amarilla. Antes de su clausura se destruyó la arquitectura, como banquetas y muros reduciéndolos hasta sus bases. 
Etapa Tardía: constituye el último momento de ocupación en la cima de la pirámide, siendo la etapa de la que se ha obtenido mayor información del diseño arquitectónico de la sexta plataforma. Los muros de esta etapa llegan a tener el menor ancho registrado en los muros de Huaca Pucllana, variando entre $0.45 \mathrm{~m}$ a $0.50 \mathrm{~m}$, los pisos muestran poca elaboración y se registra eventos de clausura de los espacios (Ccencho, 2015; Paz, 2015; Ganoza, 2015); cabe mencionar que el estado de conservación de dichos elementos arquitectónicos no es muy bueno, los pisos presentan varias alteraciones e intrusiones post abandono - prehispánico y contemporáneo, de igual manera los muros se encontraron reducidos hasta sus bases. Está etapa se compone de nueve ambientes: dos de los cuales son patios con banquetas adosadas; un patio parcialmente excavado; tres recintos de dimensiones menores, los cuales presentan acceso que los comunican entre sí; y tres pasadizos, dos de los cuales se orientaron de Norte a Sur y uno de Este a Oeste. Estos pasadizos se encuentran en torno a los patios con banquetas y otro con acceso a estos ambientes. Cabe mencionar que los muros excavados se encontraban reducidos hasta sus bases (conservaban una altura máxima de $0.16 \mathrm{~m}$ ), si bien se dió por el desmantelamiento de la arquitectura al momento del abandono, también se debió a la exposición que tuvo durante todos los años de abandono, asimismo los pisos presentaron roturas, y grietas; los hoyos intrusivos afectaron tanto a pisos como a muros.

En la planimetría se puede apreciar que se trató de un sistema que involucra pasadizos que llevan a patios grandes y patios con banquetas y asociados a recintos de menor tamaño (Figura 7).

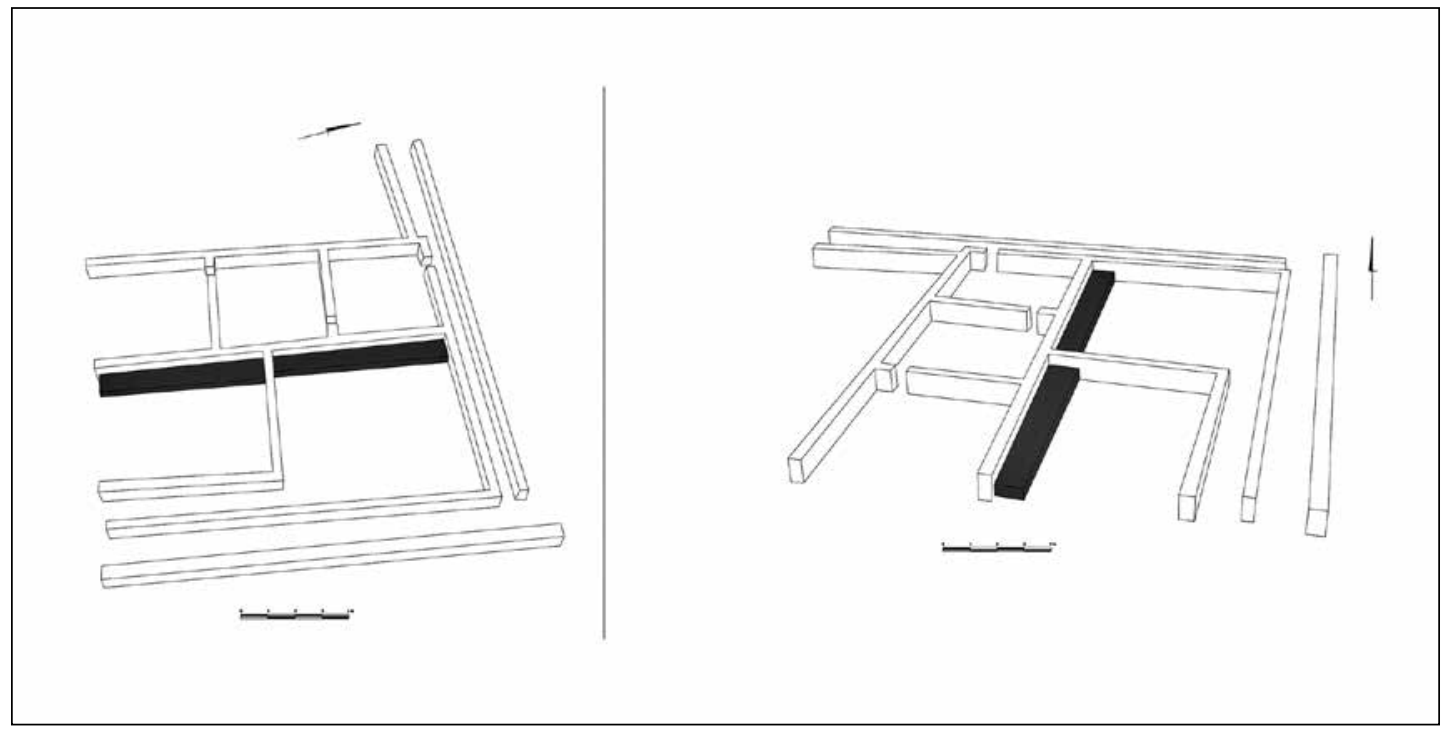

Figura 7.

La UA-03(13) es un pasadizo de eje Este-Oeste, este pasadizo se ensancha desde el Este donde tiene un ancho de $0.7 \mathrm{~m}$ para luego tener en el extremo Oeste un ancho de $1.2 \mathrm{~m}$. Está flanqueado por el norte por el muro MU-38(09) y por Sur con los muros MU-37(09) al que se le adosa en su extremo Oeste el muro MU-07(13). De manera tardía se abrió un acceso en el muro MU-37(09), retirando solo algunos adobes originando un vano de $0.6 \mathrm{~m}$ de ancho, permitiendo el acceso a la UA-04(13) desde este pasadizo. El piso [PI-04(14)] se encuentra muy desgastado debido al uso que debió tener; al finalizar el tiempo de uso del pasadizo se cubrió con un depósito de tierra con restos vegetales como maíz, carrizo, etc. y luego relleno de adobes. 
La UA-05(13) se encuentra en el extremo Este de la sexta plataforma, teniendo como limite la ladera este; es un espacio amplio del tipo patio. Este patio limita por el Oeste con la ladera de la pirámide, al Norte se encuentra el muro MU-07(13) y el pasadizo UA-03(13), y al Oeste el muro MU-06(13). Tuvo dos pisos asociados al uso del espacio, el más temprano es el piso PI-09(14) y el más tardío el piso PI10(14) que se encontró muy desgastado y agrietado, parte del piso fue cubierto con una capa de barro batido con restos de material orgánico y relleno de adobes.

La UA-04(13) se encuentra al Este del patio UA-05(13) y sur del pasadizo UA-03(13); es un recinto pequeño de forma trapezoidal el cual tiene dos accesos ubicados en sus muros norte y sur, está formado por los muro MU-37(09) al norte, MU-36(09) al Este, MU-01(14) al Sur y el MU-06(13) al lado Oeste; todos los muros presentan enlucido de barro de color beige y $5 \mathrm{~mm}$ de grosor. El piso PI-01(14) se encontró muy desgastado y con hoyos de base cóncava y huellas de restos vegetales, como si en algún momento estuvo la superficie muy húmeda, pudiendo relacionar esto con la posible actividad del recinto: servicio/consumo de líquido (Paz, 2015: 212). También se encontró cubriendo el piso: capas de barro mezclado con abundante material orgánico, y algunos lentes de ceniza. Se comunicó directamente con la UA-01(14) y la UA-03(13) por los vanos de accesos registrados, los dos con eje Norte-Sur; al momento del abandono los vanos fueron sellados con adobes y argamasa de barro.

La UA-01(14) se encuentra al Este de la UA-05(13), al Sur de UA-04(13); es un recinto pequeño de forma cuadrangular, similar al descrito anteriormente, presenta dos vanos de acceso en los muros norte y sur; está formado por los muros: MU-06(13) por el Oeste, MU-01(14) por el norte, MU-36(09) por el Este y el MU-02(14) por el Sur. El piso PI-06(14) se encuentra desgastado y como actividad para el abandono se encontraron concentraciones de cerámica (Lima y alfar Pucllana Nievería), también en el extremo norte del recinto una capa de tierra mezclada con abundante restos vegetales y otros materiales como restos malacológicos, cráneos de cuyes; finalmente cubriendo parte de esta capa se colocó una capa de barro batido y sellaron los vanos de acceso.

La UA-02(14) se encuentra al Este de UA-05(13), Sur de UA-01(14) y Oeste de UA-01(12); es un recinto similar a los descrito anteriormente, tiene comunicación directa por el vano de acceso en el muro norte; tendría una forma cuadrangular y está formado por los muros: al Este el muro MU-06(13), al Norte el muro MU-02(14) y al Oeste el muro MU-36(09). El piso PI-07(14) se encontró desgastado y en este recinto se da lo contrario que en el anterior, para cubrir el piso primero colocan una capa de barro en el extremo Oeste del ambiente, luego se encuentran lentes de la capa de tierra mezclada con abundantes restos orgánicos, además colocaron sobre el piso una ofrenda de maíz, la cual estuvo envuelto en un tejido llano (muy deteriorado), a diferencia del recinto anterior en este se encontraron abundantes restos óseos de aves pequeñas y fragmentos de antara de arcilla.

La UA-01(12) se encuentra al Este de las UA-01(14) y UA-02(14), al Sur de la UA-02(09) y al Oeste de la UA-02(12); se trata de un patio con banqueta de planta rectangular, se encuentra parcialmente excavado. La banqueta BA-01(14) se encuentra adosada al muro Oeste MU-36(09) y estaría orientada de Sur a Norte; tiene un ancho de $1.22 \mathrm{~m}$. El piso PI-05(14) se encontró desgastado, en este recinto se definió al menos tres momentos de ocupación. De manera tardía se elaboró una estructura paralela a la banqueta (se desconoce su uso y forma original) la cual estaba formada por un murete de adobes colocados verticalmente y una hilera de cañas de carrizo frente a este murete, tuvo un ancho de $1.2 \mathrm{~m}$ y en su interior se encontraron objetos elaborados en concha nácar (Flores, 2013a: 108).

La UA-02(09) se encuentra al Este de las UA-04(13) y UA-01(14), al Norte de UA-01(12), Sur de UA03(13) y Oeste de UA-09 (12); se trata de un patio con banqueta (de mayor tamaño que la anterior), tuvo una planta cuadrangular y conserva principalmente el lado Oeste, donde estuvo la banqueta 
adosada al muro MU-36(09). El piso del recinto PI-02(12) es muy delgado y presentó mucho desgaste. $\mathrm{Su}$ acceso se encontró hacia la esquina Sureste que termino luego en el pasadizo de la UA-09(12). En la parte media del recinto, se encontró en el piso improntas de vasijas alineadas.

La UA-09(12) se ubica al Oeste de la UA-02(12) y la UA-02(12); se trata de un pasadizo de orientación Norte - Sur, Este pasadizo tiene un ancho de $1.40 \mathrm{~m}$. El piso que se ha conservado PI-10(12) y se encontró muy desgastado. Está formado por el muro MU-15(12) ubicado al Este y al Oeste el MU-07(12), ambos se encontraron en mal estado de conservación.

La UA-02(12) se ubica al Sur de la UA-01(12). Corresponde a un pasadizo con dirección Norte-Sur que conduce a la UA-02(09), debe de prolongarse hacia el Sur (área no excavada). El pasadizo tiene un ancho de $1.50 \mathrm{~m}$, del cual solamente se pudo encontrar una pequeña área. Está definido por los muros MU-02(12) ubicado en el lado Oeste, el que forma esquina con el MU-01(12), este muro tiene solo $0.45 \mathrm{~m}$ de ancho; hacia el lado Este se ubica el MU-07(12), el cual se encontró muy mal conservado y destruido; el piso del pasadizo es PI-08(12), el cual se encuentra muy desgastado y en mal estado de conservación.

La clausura de los espacios para el abandono definitivo de la sexta plataforma se dio con el siguiente proceso:

- Se realiza destrucción de los espacios, principalmente por remoción de los hoyos de poste o realización de hoyos.

- Se dejan depósitos de tierra mezclados con abundante restos de orgánicos (principalmente vegetales) sobre el piso, ofrendas de maíz, concentraciones de cerámica (Lima y Nievería).

- Se pasa a cubrir parte de estas acumulaciones con barro compacto5.

- Se sellan los vanos de acceso colocando adobes con poca argamasa de barro.

- Se cubren los espacios con un relleno de adobes enteros y fragmentados, además se destruye la arquitectura, los muros son destruidos hasta sus bases.

- Abandono definitivo del Centro Ceremonial.

En conclusión y sobre lo observado en los diferentes ambientes descritos, los espacios van cambiando su diseño arquitectónico dentro de cada etapa arquitectónica, desde una plaza amplia, hasta llegar a un sistema restringido de patio y patios con banqueta con recintos menores asociados, a los que se accede por medio de pasadizos largos y estrechos. Esto podría estar indicando cambios en la dinámica social de la sociedad Lima, que culminó con el abandono definitivo del centro ceremonial.

\section{Sobre el Material Cerámico Asociado}

Siendo el alfar Pucllana Nievería el eje de la cronología relativa para la ocupación de la Fase constructiva IV, sobre esta ya se ha señalado que no solo coexiste con los demás alfares registrados en Pucllana, además se puede diferenciar temporalmente en dos épocas: alfar Pucllana Nievería temprano, con antecedentes en la cerámica Pucllana Naranja, siendo el inicio del estilo Nievería, el cual se relaciona más con la iconografía de la costa Sur que de la sierra; y alfar Pucllana Nievería tardío ${ }^{6}$ que se diferencia del anterior por incorporar el color gris y motivos decorativos que se vinculan con diseños ayacuchanos (Ccencho, 2006: 33). Asimismo la relación estratigráfica del alfar Pucllana Nievería temprano se asocia las construcciones de la Fase Constructiva III - crecimiento de la huaca; mientras que las últimas ocupaciones hasta el abandono del sitio ya se asociaba al alfar Pucllana Nievería tardío (Ccencho, 2006:31, 32). 
La cerámica de alfar Pucllana Nievería de esta investigación se recuperó de rellenos arquitectónicos de remodelación y clausura, así como de desechos de basura sobre los pisos de los recintos, hoyos y hoyos de poste; la cerámica Nievería temprano se encuentra presente en las tres etapas de la Fase Constructiva IV, sin embargo el Nievería tardío se asocia más a la clausura de la última etapa de ocupación, pero coexistiendo con los otros alfares Pucllana.

En la muestra de cerámica recuperada se obtuvo una tendencia ya observada en Huaca Pucllana (Ccencho, 1999). La mayor presencia la tiene el alfar Pucllana Naranja, siendo predominante no solo la muestra general sino en cada etapa constructiva; en segundo lugar corresponde al alfar Pucllana Marrón, seguido por gran diferencia el alfar Pucllana Nievería, Pucllana Nievería Gris, alfar Pucllana Gris, y alfar Pucllana Fino.

En la Etapa Temprana el material cerámico analizado procede de pisos, hoyos de poste y rellenos de clausura, ésta determinó que la mayor cantidad de fragmentos corresponden al alfar Pucllana Naranja, seguido del alfar Pucllana Marrón y de Pucllana Gris, siendo escasos los alfares Pucllana Fino y Pucllana Nievería, el Pucllana Nievería Gris está ausente. En cuanto a las formas, las vasijas cerradas son las numerosas, sobre todo las ollas y las vasijas abiertas más predominantes son los platos. En cuanto a la decoración en las vasijas abiertas se tienen diseño tanto geométricos (bandas y líneas: verticales y horizontales) como figurativos (seres entrelazados de cabeza triangular). De las vasijas cerradas se tiene decoración del tipo punteada/incisa en un cántaro de alfar Pucllana Gris.

En la Etapa Media el material cerámico proviene de pisos, hoyos de poste, desmontaje de la banqueta, acumulación de desechos sobre el piso y del relleno de clausura; esto mostró mayor presencia del alfar Pucllana Naranja, por encima de los demás alfares, se observa la presencia de fragmentos del alfar Pucllana Nievería y ausencia del Pucllana Nievería Gris. En cuanto a las formas, la mayor cantidad corresponde a vasijas cerradas, en especial ollas y cántaros; las vasijas abiertas están representadas por platos (de alfar Pucllana Nievería y Pucllana Naranja), pero en mucha menor cantidad. En cuanto a los diseños, predominan los motivos geométricos de banda y líneas (verticales y horizontales) y distintas formas geométricas como triángulos delineados, principalmente en colores negro y blanco, aunque se observa la presencia del color rojo en algunos motivos geométricos; los motivos figurativos representan principalmente al ser entrelazado, pero solo se encuentra en pocos fragmentos en delineado negro y del estilo blanco sobre negro (Ccencho, 1999: 146).

En la Etapa Tardía se obtuvo mayor cantidad de contextos analizados, estos provienen principalmente de las acumulaciones de desechos sobre los pisos, capas de barro, concentraciones de cerámica y rellenos de clausura. Se observó que el alfar Pucllana Naranja es el más frecuente, seguido del alfar Pucllana Marrón, les sigue el alfar Pucllana Nievería y Pucllana Nievería Gris, por encima de los alfares Pucllana Gris y Pucllana Fino, los cuales son escasos en esta etapa. En cuanto las formas más predominantes son las vasijas cerradas, observándose ollas, cantaros y una botella de alfar Pucllana Nievería; las vasijas abiertas no son tan escasas como en las etapas anteriores, teniendo platos de alfar Pucllana Naranja, mayormente sin decoración y curiosamente se tiene platos del alfar Pucllana Nievería Gris, estos se caracterizan por tener una pasta muy fina, la superficie pulida casi bruñida, uno de ellos tiene decoración en el labio, de la cabeza de un ave, también se tiene un fragmento de plato/cuenco el cual tuvo la forma de una lagenaria y el otro perteneció a un contexto de concentración de cerámica encontrado sobre el piso de un recinto, pertenecería a una clase de plato que tendría la particularidad de tener la base hueca o un espacio vacío en la base. (Figura 8 ) 


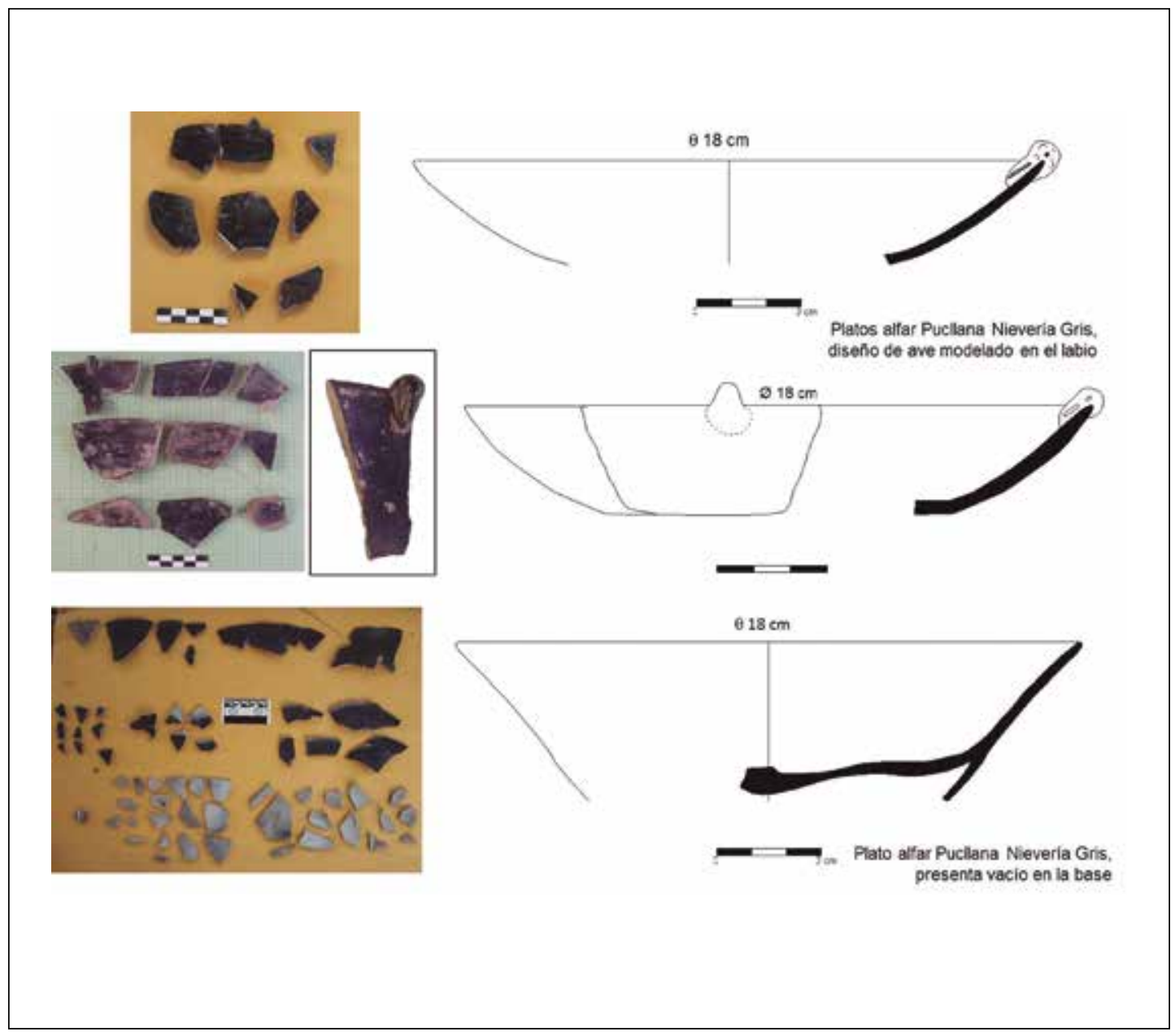

Figura 8.

Los diseños encontrados corresponden a motivos geométricos y figurativos ya conocidos en el repertorio Lima, para los fragmentos de alfar Pucllana Nievería se ha podido identificar diseños que aluden al pulpo delineado negro sobre fondo rojo oscuro y parte de un ojo pintado de blanco y delineado con negro acompañado de un panel de color gris, además de argollas delineadas en negro y bandas negras delimitando paneles. (Figura 9). De lo descrito, los alfares propios de la sociedad Lima, como es el característico alfar Pucllana Naranja, continuaron tanto en sus formas como en sus motivos decorativos, correspondiendo al Lima Tardío (Figura 10). A pesar de la poca fragmentería recuperada de los alfares Pucllana Nievería y Pucllana Nievería Gris, su presencia resulta innegable para observar los cambios relacionados al grupo social que habitó en Pucllana y se encargó de las construcciones correspondientes a la Fase Constructiva IV, el diseño del pulpo y la presencia del color gris lo asocia a Pucllana Nievería Tardío del Horizonte Medio 1B; sin embargo el motivo de ave modelada en el labio del borde de un plato de alfar Pucllana Nievería Gris posiblemente mostraría el apego a sus tradiciones costeñas, además de los productos agrícolas costeños por la presencia de un plato con forma de lagenaria. 

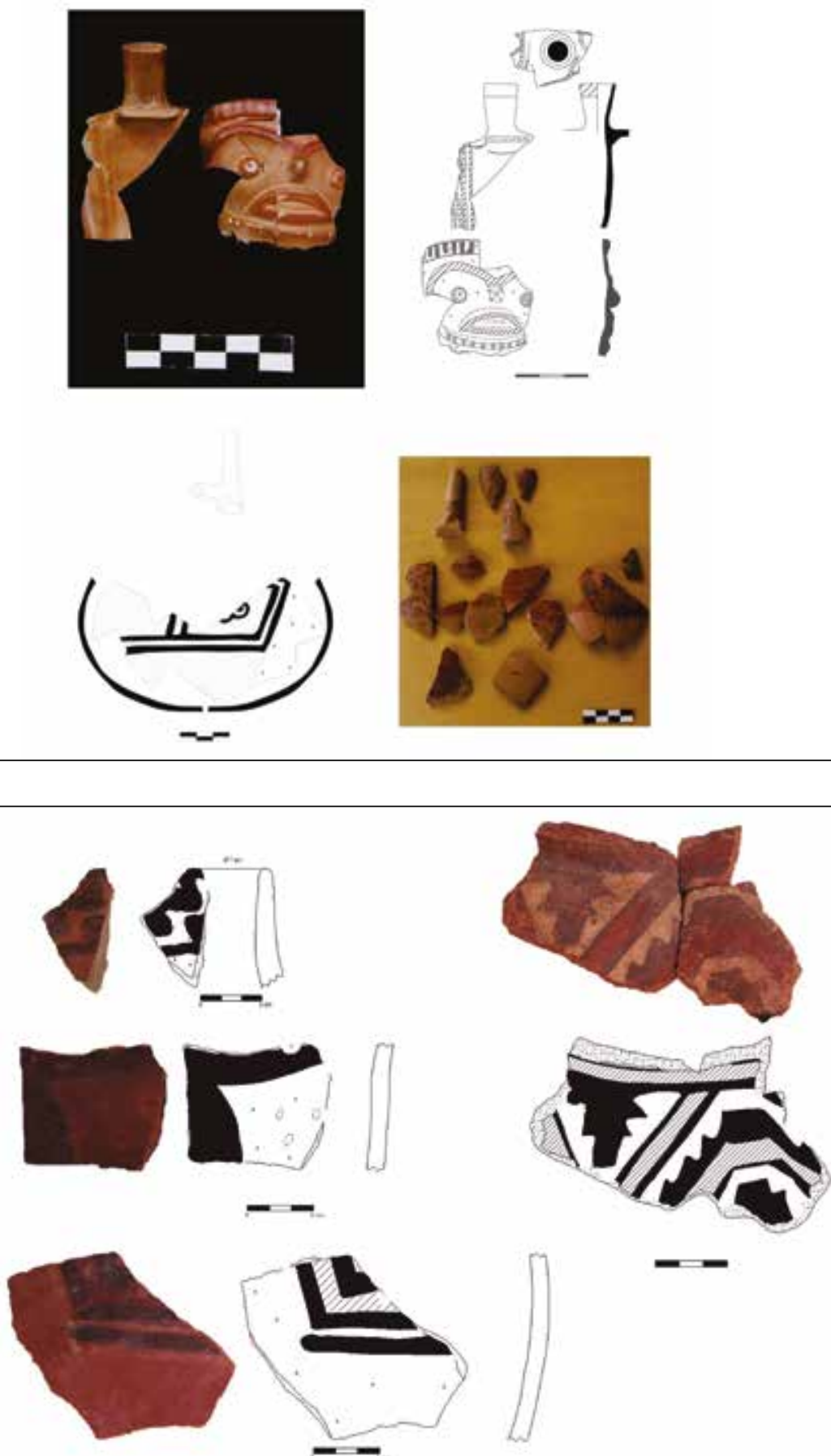

Figura 9. 


\section{Comentarios Finales}

Los datos obtenidos en las excavaciones en Huaca Pucllana muestran que el desarrollo arquitectónico fue continuo desde el periodo Intermedio Temprano hasta la primera época del Horizonte Medio. Es durante la época 1A del Horizonte Medio que se evidencian los primeros cambios relacionados a lo ideológico y político, manifestado en el crecimiento máximo del centro ceremonial, remodelación de los espacios construidos, refacciones de pisos, etc., y caracterizados por la presencia del alfar Pucllana Nievería temprano (Ccencho, 2006:31; Nilton y Ccencho, 2009:105). En la época 1B del Horizonte Medio se define por la presencia del alfar Pucllana Nievería Tardío, el cual tiene influencias foráneas en el diseño, formas y el aumento de colores, esto sumado a los cambios internos ocurridos en Pucllana que han sido reflejados en la arquitectura, hasta llegar al abandono del sitio (Ccencho, 2006: 31). Se cuenta con un fechado ${ }^{7}$ que coloca el inicio de la Fase Constructiva IV - Etapa Temprana en el 600 d.C, es decir en el periodo Horizonte Medio época 1B (Flores, 2015). Creemos que esta fase constructiva fue planeada y sacralizada por la presencia de dos sacrificios humanos en un muro del extremo sur de la quinta plataforma (Barreto et al. 2010).

Los cambios ocurridos en la sexta plataforma de Huaca Pucllana previos al abandono del sitio habían sido poco tratados, es gracias a las últimas excavaciones realizadas que se ha podido vislumbrar un panorama más amplio, mostrando no solo que la presencia de la cerámica con el alfar Pucllana Nievería Tardío se encuentra relacionada a estos cambios drásticos de la arquitectura, sino que estos mismos estarían conformando una fase constructiva, que fue dividida en tres etapas. La arquitectura de esta fase presenta marcadas diferencias en sus rasgos morfológicos y tecnológicos lo que la separaba de la fase constructiva anterior. Estos cambios debieron originarse por "las transformaciones sociales que se estaban generando en la cada vez más compleja sociedad Lima" (Nilton y Ccencho, 2009: 115) y que culminó con el posterior abandono del centro ceremonial.

Llama la atención el desapego a algunas prácticas como la ausencia de pintura amarilla en las paredes de los recintos, mostrando probablemente poco de interés en representar ese aspecto religioso, ya que el grupo dominante (digamos clase sacerdotal) pasaba por un desprestigio que fue manifestado en la arquitectura, pero resulta innegable que las formas arquitectónicas, como los patios con banquetas (espacios de actividad ritual para un grupo determinado de la sociedad), concentraciones de cerámica rota y acumulaciones de desechos sobre los pisos, entre otros, siguen presentes posiblemente como continuidad de la identidad Lima. Para el final de la ocupación de la Fase Constructiva IV - Etapa Tardía, se realiza actividades rituales que culminan con el sello de los recintos y desmantelamiento de los elementos arquitectónicos para luego retirarse por completo del centro ceremonial.

Luego del abandono de Huaca Pucllana, se sabe que otros sitios de la sociedad Lima continuaron siendo ocupados, como es el caso de Huaca San Marcos, Cajamarquilla, Pachacamac, entre otros; estos muestran mayor presencia y/o influencia Wari representado en la cerámica con el estilo Pachacamac (Shady, 1988; Chauca, 2009). Cabe mencionar y resaltar que no se ha registrado en Huaca Pucllana ocupación arquitectónica relacionada a la sociedad Wari, ésta tiene presencia en el sitio cuando ya se encontraba abandonada y fue usado como "cementerio de élite" (Flores, 2005; 2013b; 2013c; Flores et al. 2012a).

Por último, esta propuesta debe ser complementada y/o puede llegar a ser modificada a medida que avanzan las investigaciones en Huaca Pucllana. 


\section{AgRAdecimientos}

Agradezco a la Dra. Isabel Flores por la oportunidad y la confianza brindada en la participación de las excavaciones y la realización de esta investigación. Un agradecimiento especial a los colegas PICPVHP por sus contribuciones en el tema (durante la excavación y los análisis de materiales), por último a los estudiantes de arqueología de la UNMSM que ayudaron en el análisis y dibujos de cerámica.

\section{Notas}

1 Se trata de dos contextos funerarios: CF-03(07) de sexo femenino, se ubicó 30cm por encima del CF-05(09) de sexo masculino; de este último se tomó una muestra para fechado radiocarbónico (muestra procedente de diente del individuo).

2 J. Ccencho (2006: 19) menciona que se debe distinguir del alfar Pucllana Gris, ya que el alfar Pucllana Nievería Gris tendría la misma pasta que el alfar Nievería, pero elaborado en cocción reductora, formando un nuevo alfar diferenciado.

3 Los contextos excavados son identificados con dos letras y un número correlativo de acuerdo a su naturaleza (piso, muro, banqueta, unidad arquitectónica, etc.) y a su vez con los dos últimos números de la temporada de excavación entre paréntesis; ej. MU-08(12).

4 Esta actividad compromete la participación de numerosas personas y parece ser una ceremonia de carácter abierto, probablemente se trate del pueblo ofrendando, de ahí el carácter reducido de las ofrendas y el gran número de hoyos (Flores, 2005:83).

5 Esto puede variar, colocando primero y en una zona del recinto la capa de barro y luego la acumulación de desechos orgánicos, como sucede en los recintos UA-01(14) y UA-02(14) (Recintos pequeños).

6 Esta cerámica se caracteriza principalmente por tener diseños que aluden al pulpo, además de argollas y paneles delineados en negro, también se da la presencia del color gris, como principales indicadores

7 Fechado radiocarbónico: Muestra Oxa - 31118. Laboratorio de la Universidad de Oxford, calibrado en programa Oxcal v.4.2.

\section{BibliografíA}

BARRETO, María; CCENCHO, José; SILVERA, Hernán y CHUCHÓN, Hilda

2010 "Sacrificios humanos Lima asociados a la última etapa constructiva de Huaca Pucllana". Arqueología y Sociedad N² 22: 55 - 72. Museo de Arqueología y Antropología, Universidad Nacional Mayor de San Marcos. Lima.

CCENCHO, José

1999 “Alfarería Pucllana: Propuesta de una metodología de clasificación y algunos aportes para el entendimiento de la cultura Lima". XII Congreso peruano del hombre y la cultura andina "Luis G. Lumbreras" Tomo II. Ismael Pérez, Walter Aguilar y Medardo Purizaga Editores. Universidad Nacional San Cristóbal de Huamanga. 140 - 151. Ayacucho. 
2006 "El alfar Pucllana Nievería. Cambios registrados en una vajilla ceremonial y sus implicancias sociales". Cuadernos de Investigación/INC Arqueología № 1: 17 - 34. Instituto Nacional de Cultura. Lima

2012 "Algunas contribuciones para el entendimiento de la vajilla Alfar Pucllana marrón doméstica Lima”. Revista del Instituto de Investigaciones Sociales Nº 28: 321 - 332. Universidad nacional Mayor de San Marcos. Lima.

2015 Excavaciones en el lado Noreste de la sexta plataforma de la Gran Pirámide de Huaca Pucllana. Informe Final de Excavación - Temporada 2014. Isabel Flores (2015). Ministerio de Cultura. Lima.

CHAUCA, George

2009 Uso y abandono de la plataforma 2 de Huaca San Marcos durante la época 2 del Horizonte Medio. Tesis para optar el título de Licenciado en Arqueología. Universidad Nacional Mayor de San Marcos. Lima.

FLORES, Isabel

2005 Pucllana: esplendor de la Cultura Lima. Perú. Instituto Nacional de Cultura.

2013a “Informe final de las excavaciones del Proyecto Arqueológico Huaca Pucllana - Temporada 2012”. Informe presentado al Ministerio de Cultura.

2013b Huaca Pucllana y la ciudad de Miraflores: El antiguo origen de un moderno distrito. Lima. Editorial Argos.

2013c Los Wari en Pucllana. La tumba de un sacerdote (compiladora). Lima. Ministerio de Cultura.

FLORES, Isabel; CHUCHÓN, Hilda; CCENCHO, José y VARGAS, Pedro

2012a La doble tela de los muertos: tejidos especiales Wari de Huaca Pucllana. Lima. Auqi Ediciones.

FLORES, Isabel; VARGAS, Pedro; CCENCHO, José y SILVERA, Hernán

2012 b "Los patios con estructuras escalonadas de Huaca Pucllana: Caracterización y función de arquitectura ceremonial Lima". Arqueología y Sociedad № 25: 57 - 88. Museo de Arqueología y Antropología, Universidad Nacional Mayor de San Marcos, Lima.

GANOZA, Mirella A.

2015 Excavaciones en el área Norcentral de la sexta plataforma de la Gran Pirámide de Huaca Pucllana. Informe Final de Excavación - Temporada 2014. Isabel Flores (2015). Ministerio de Cultura. Lima.

MAÑANA, Patricia; BLANCO, Rebeca y Ayán, Xurxo M.

2002 “Arqueotectura: Base teórico metodológica para una arqueología de la arquitectura”. TAPA 25. Laboratorio de Patrimonio, Paleoambiente e Paisaxe. España. Instituto de Investigacións tecnolóxicas, Universidad de Santiago de Compostela.

MAQUERA, E.; ESTEBAN, M.; ÁLVAREZ, M.; GÓNZALES, L. M.; PEDEMONTE, V.; SILVA, P.; PAZ, Gladys

2015 "Arquitectura vida y muerte en Lima entre los años 600 a 1000 d.C. Nuevos alcances desde la zona arqueológica Catalina Huanca”. Arkinka, N² 234: 92-101. Lima. 
MOORE, Jerry

1996 "Architecture and the Power in the Ancient Andes". The archaeology of public building. United Kingdom. Cambridge University Press.

PAZ, Gladys

2015 Excavaciones en la esquina Noroeste de la sexta plataforma de la Gran Pirámide de Huaca Pucllana. Informe Final de Excavación - Temporada 2014. Isabel Flores (2015). Ministerio de Cultura. Lima.

Ríos, Nilton

2008 Restos de actividades rituales en la segunda etapa constructiva de una plaza Lima Tardío: un caso en Pucllana. Tesis para optar el título de Licenciado en Arqueología (2 tomos). Universidad Nacional Mayor de San Marcos. Lima.

RÍos, Nilton y Ccencho, José

2009 "Cambios en la sociedad Lima reflejados en la arquitectura del centro ceremonial Pucllana durante las primeras épocas del Horizonte Medio. Las evidencias de la Plataforma IV". Arqueología y Sociedad N²0: 91-118. Museo de Arqueología y Antropología, Universidad Nacional Mayor de San Marcos. Lima.

SILVERA, Hernán

2012 "Buscando rastros de una actividad ritual en Huaca Pucllana". Revista del Instituto de Investigaciones Sociales $\mathrm{N}^{\circ}$ 28: 313 - 320. Universidad Nacional Mayor de San Marcos. Lima.

SHADY, Ruth

1982 “La Cultura Nievería y la interacción social en el mundo andino en la época Huari”. Arqueológicas $\mathrm{N}^{\circ}$ 19: 5-82. Lima.

1988 “La época Huari como integración de las sociedades regionales”. Revista Andina, año 6, № 1: 67 - 133. Lima.

VARGAS, Pedro

2012 “Secuencia constructiva de la Gran Pirámide de Huaca Pucllana en sus tiempos finales". Revista del Instituto de Investigaciones Sociales - Investigaciones Sociales $\mathrm{N}^{\circ} 28: 303$ - 312. Universidad Nacional Mayor de San Marcos. Lima. 